\title{
Validations of a Model to Estimate Thermal Conductivities of Ceramics
}

\author{
Bashir M. Suleiman \\ Department of Applied Physics and Astronomy, College of Sciences, University of Sharjah, Sharjah, United Arab Emirates
}

Email address:

bashir@sharjah.ac.ae, b.suleiman7@gmail.com

\section{To cite this article:}

Bashir M. Suleiman. Validations of a Model to Estimate Thermal Conductivities of Ceramics. Advances in Materials.

Vol. 5, No. 5, 2016, pp. 44-50. doi: 10.11648/j.am.20160505.13

Received: September 6, 2016; Accepted: September 18, 2016; Published: October 11, 2016

\begin{abstract}
The Effective Medium Theory Model (EMTM) used in this work is based on the assumption that every phase of the matrix is embedded in a homogeneous medium with conductivity $k_{\text {eff }}$ to be determined self-consistently. It is based on dilute spherical inclusions of one phase embedded in a matrix of a second phase. Several Samples of composite ceramics that are mechanically strong, relatively non-porous and anisotropic have been investigated. A comparison between the measured data and the results predicted by EMTM were made to validate the model for these ceramic samples. In particular, we investigate the effect of mineralogy (constituents) in ceramics and their spatial distribution profile to validate the homogeneity conditions of the model. Preliminary indicators of validation were used to check the bulk and surface homogeneities. This can be done either by roughly estimating Wiener bounds or by examining microscopically the surfaces of the samples. It turns out that the EMTM is a suitable one to estimate $k_{\text {eff }}$ provided that the homogeneity conditions are satisfied.
\end{abstract}

Keywords: Thermal Conductivity, Effective Medium Theory, Ceramics, Composites

\section{Introduction}

Composite ceramics differ from traditional materials, it is the combination of two or more materials in which composite parts comprise at least two distinctly different components mixed and bonded on macro and microscopic scales. These materials are promising materials, which should exhibit an improve on several aspects of the physical properties such as mechanical, thermal, electrical etc. Their properties will depend on the morphology (Phase distribution), the microstructure features and the required design to satisfy the dynamic expected behavior of the desired application $[1,2]$.

There are numerous applications to use composite materials to enhance the thermal performance. For example, $\mathrm{Al}_{2} \mathrm{O}_{3}$ and Aluminum Nitride Ceramic Substrates (AIN) are used as substrates for integrated circuits in the field of electrical engineering for electrical insulation, and heat conductivity in electronics. Another example, among various refractory materials, $\mathrm{SiC} / \mathrm{SiC}$ ceramic matrix composites (CMC) are of prime interest for fusion and advanced fission energy applications, due to their excellent irradiation tolerance and safety features (low activation, low tritium permeability,...). These examples are some of the concrete evidences that thermally related applications either in fission energy or heat conductivity in electronics [3].

In order to use models to calculate the effective thermal conductivity of composite solid materials, it requires the previous knowledge of several parameters that are crucial to the calculation process. These parameters are including but not limited to the thermal conductivity of each component, the volume concentrations of the component, the component morphology (distribution), the shape of inclusions of the different components in the solid matrix, and the fractional porosity if the medium is porous $[4,5]$.

Thus, in general, the theoretical models to calculate the thermal conductivity of composites will depend on the following three factors: (i) intrinsic microstructure features (mineralogy) of all phases. (ii) the spatial distribution of each phase and (iii) the dynamic behavior of the desired application [6].

\subsection{Microstructure Features}

The selection of various composite materials that are 
suitable for diverse applications $[7,8]$ can be achieved due to the distinctive character of the detailed microstructure features of the composites. It is possible to fine-tune the material microstructure with small modifications of sizing of the strengthening items (reinforcement) such as particles, flakes, fiber volume, and resin additives and so on, to develop the desired material and property. For example, composites can make up the bipolar plates, end plates as in the case of system components of proton exchange membrane (PEM) fuel-cell systems. Chopped carbon fiber and graphite particle-filled/vinyl ester bulk molding compounds (BMCs) are finding wide use in bipolar plates for low-temperature PEM fuel cells. Another example, wing leading edges in missile technology requires low fiber volume in these thin structures to reduce edge's erosion

Furthermore, micro-material testing systems offer position measurement resolution of better than $20 \mathrm{~nm}$ which encourages the selection of composites over traditional materials such as metal alloys or plastics. This will lead to optimization in the desired properties regarding size, cost, weight, surface condition, thermal/electrical conduction and mechanical strength. A relevant example to this work is $\mathrm{SiC} / \mathrm{SiC}$ ceramic matrix composites (CMC) that have Effective thermal conductivity $\left(\kappa_{\text {eff }}\right)$ value of $\sim 320 \mathrm{~W} / \mathrm{m}-{ }^{\circ} \mathrm{C}$ at room temperature higher than the thermal conductivity of some precious expensive metals [8].

\subsection{The Spatial Distribution of Phases}

To illustrate this factor, if we consider a block of solid composite consisting of layered slabs of different components (phases) will give a maximum effective conductivity in the parallel direction (Parallel distribution of phases) and a minimum effective conductivity in the perpendicular direction (Serial distribution of phases). These two extreme results (bounds) were first introduced by Wiener [9]. According to the definitions of Wiener bounds, a dry composite sample consisting of a mixed orientation of components layers (parallel and serial distributions of phases), is expected to have an effective conductivity value within these two bounds. More details are given elsewhere [10].

\subsection{Dynamic Behavior}

There is a class of composites which are characterized by dynamic behavior and have peculiar compositional and microstructural variation in space. A good example of this type is known as Functionally Graded Materials [6, 11-13]. FGMs are really complex systems: they are multi-phase materials, with functionally graded fibrous composites. Their dynamic effective thermal behavior and the volume fraction of the materials changes gradually. They are innovative composite materials whose composition and microstructure vary in space following a predetermined law. The gradual change in composition and microstructure gives place to a gradient of properties and performances
[11]. It is the non-homogeneous microstructures in these materials that produce continuous graded macroscopic properties, such as the thermal conductivity, specific heat, mass density and elastic modulus. FGM's have been developed as the super-resistant materials for propulsion systems and airframe of the space planes in order to decrease thermal stresses and to increase the effect of protection from heat [14-15]. FGM's can reduce the thermal stress in such structures working in high temperature environment. All the effective thermal properties of FGMs can provide great help in predicting the overall behavior under various loading conditions [14]. The least to say is that the theoretical and experimental investigation of the effective thermal properties of all composites including FGMs and the role of interfaces in composite materials are areas which have received great interest in recent years. For example, a recent review article by Cahill et al. [16] discusses the effect of interfaces in composite materials that involve nanoparticle suspensions. In this way, the interfacial thermal transport may involve a complicated process in which the phonons carrying most of the heat across the interface are not necessary the phonons carrying the heat in the bulk or along the fiber, which in turn makes phonon-phonon coupling within each material forming the interface an important factor. This will require more advances in experimental techniques to provide improved control and characterization of interface structure.

In this work, a comparison between the measured data and the results from predictions of theoretical models has been made for several composite ceramic solid samples excluding nanoparticle suspensions.

\section{Background Features of the Theoretical Models}

Theoretical investigations to estimate the effective thermal conductivity of composites have been active since 1892 when Maxwell proposed his Model [17]. The mathematical expression for this model was based on the simplest form of two-phase dispersion containing spherical particles of the first phase (material) imbedded into a continuous medium of a second phase (material), neglecting the interactions between the particles. The model validity was limited to very low concentrations of the first phase i.e. for very dilute volume fraction. Later Rayleigh [18] proposed a second model including the effect of interactions when the concentrations of the spherical particles are large and the effect cannot be neglected. Rayleigh's model was based on considering the spherical particles as inclusions that form a cubical array, and included the interaction effect between a numbers of nearby spheres.

Other proposed works for non-spherical inclusions developed by other researchers [19-23]. Extension of these previous works was also carried out by McPhedran et al. [24] and Sangani et al. [25]. In the course of models developments, Hashin, 1968 [26] proposed a general self- 
consistent treatment. The treatment provides a physically realistic model of particle-to-particle interaction for twophase system covering the full range of the volume fraction. The self-consistent field concept is extended to include the contact resistance in the composite reinforced with coated spheres. [27]. Felske et al. [28] employed effective medium theories to predict the effective thermal conductivity of coated short-fiber composites. Later, Samantray and co-workers [29] proposed the correlations between the inclusions to estimate the effective thermal conductivity of two-phase materials. Fang et al., [13] applied the thermal wave method to investigate the unsteady effective thermal conductivity of particular composites with a functionally graded interface. A recent statistical approach has been used to estimate the effective thermal conductivity of the ensemble. This approach is associated with the variation principle that minimizes the standard deviation with optimal distribution of the parameter [30].

In general, theoretical models that are used to calculate the effective thermal conductivity of a composite material impose certain conditions and constrains that are suitable for each particular model. These conditions and constrains are directly related to the assumptions to enhance the reliability of the model and suite the expectations for the desired applications.

In this work, a comparison between the measured data and the results from predictions of theoretical models has been done for seven composite ceramic samples. The tested models include those of the effective medium theory, and Wiener bounds [9].

Within these models there are two ways to estimate $\boldsymbol{\kappa}_{\text {eff }}$, either by using exact mathematical formulas to evaluate the bounds (range) within which the conductivity must lie, or by using models based on approximate mathematical expressions.

Our selected effective medium theory model (EMTM) [31] depends on the existence of a statistically homogeneous medium surrounded by inclusions of different phases (homogeneity condition).

The procedure to validate the homogeneity conditions of the EMTM is done through preliminary indicators to check the bulk and surface homogeneities. This can be done either by roughly estimating Wiener bounds and/or by examining microscopically the surfaces of the samples through simple and preliminary microstructure surface measurements. The microscopic examination can be done by looking at the microscopic structure using other means such as point-counting method using transparent thin slices or the STM.

It should be mentioned that bounds can also be constructed in hierarchies which become narrower as the amount of structural information included grows. However, the higher order of these bounds is extremely difficult to calculate since it involves n-point correlation functions [32].

\section{Results and Discussion}

All selected seven samples are multiphase heterogeneous, mechanically strong, relatively non-porous with known volume fractions and conductivities of phases but with unknown anisotropy. The expected effective thermal conduction in these samples depends on its mineralogy (microstructure details) and the phase distribution of its constituents.

The chosen mathematical model to estimate $k_{\text {eff }}$ of the ceramic samples is based on the Effective Medium Theory (EMT). There are different formulations of EMT such as the Average Field Approximations (AFA), the Iterated Dilute Limit Approximation (IDLA), and the Coherent Potential Approximation (CPA) [32-38]. However, all are based on the assumption that every phase of the matrix is embedded in a homogeneous medium with conductivity $k_{\text {eff }}$ to be determined self-consistently. If it is assumed that the phases with $k_{i}$ 's are distributed in such a way that the material can be considered isotropic and homogeneous, and then according to EMTM, the effective conductivity $\left(k_{e f f}\right)$ is determined self-consistently by the formula:

$$
\sum_{i} P_{i}\left(\frac{\kappa_{e f f}^{M}-k_{i}}{2 \kappa_{e f f}^{M}+k_{i}}\right)=0
$$

Where, $P_{i}$ is the volumetric fraction of the $i^{\text {th }}$ component (phase).

This formula has been derived from the solution to the problem of dilute spherical inclusions of one phase embedded in a matrix of a second phase [31]. However, the formula as illustrated by Eq. (1), designates a symmetric representation to all phases without singling out a certain phase as dilute. The solution of this equation will have a physical meaning under certain imposed conditions such as satisfying $\sum_{i} P_{i}=1$ and considering $\left(k_{e f f}\right)$ as a continuance function of the volume fraction $P$.

A comparison between the measured data and the results predicted by this model has been made for the ceramic samples. In particular, we investigate the effect of mineralogy (constituents) in ceramics and their spatial distribution profile to validate the homogeneity conditions of the EMTM. Preliminary indicators of validation have been used to check the bulk and surface homogeneities. This can be done either by roughly estimating Wiener bounds or by examining microscopically the surfaces of the samples.

It turns out that the EMTM is the most suitable mathematical model to estimate $k_{\text {eff, }}$ provided that the homogeneity condition is satisfied and as required by the main assumption of the model.

The results of the selected seven heterogeneous samples of ceramics marbles and glasses are given in Table 1 and plotted in Fig. 1. The figure illustrates the $\%$ deviations from the experimental measure values, represented in the figure by the first innermost row that includes the highest columns. 


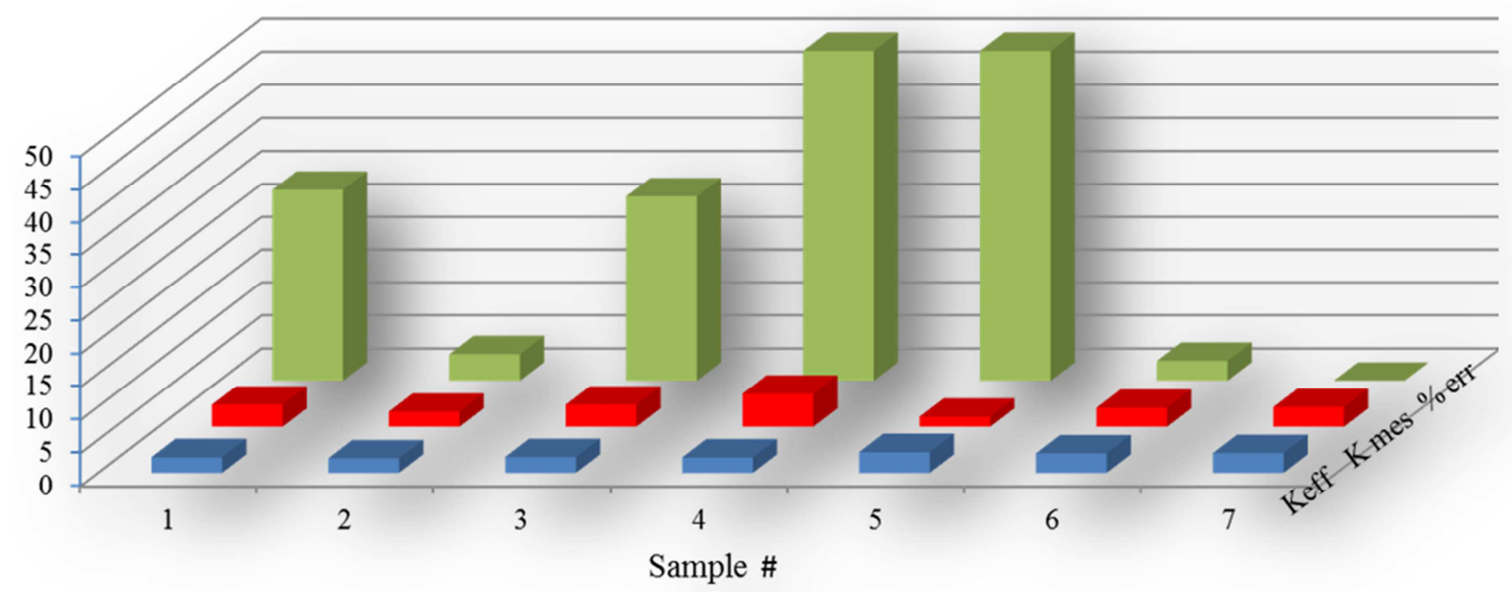

Figure 1. Comparison between the measured thermal conductivity $\left(k_{m e s}\right)$ and the calculated theoretical $\left(k_{e f f}\right)$. The calculated values and measured data along with the relative error percentage (\% error) are represented in arbitrary units by blue, red and green columns, respectively.

Table 1. The estimated $k \dot{s}[\mathrm{~W} / \mathrm{m} C]$ calculated using Wiener \& EMTT models.

\begin{tabular}{llllll}
\hline Sample & Wiener & & K eff & K mes & \% err \\
\hline \# & Parallel & Serial & & & \\
S1 & 2.59 & 2.12 & 2.36 & 3.32 & 29 \\
S2 & 2.46 & 1.96 & 2.19 & 2.27 & 4 \\
S3 & 2.61 & 2.14 & 2.39 & 3.32 & 28 \\
S4 & 2.56 & 2.06 & 2.31 & 5 & 54 \\
S5 & 8.66 & 2 & 3.12 & 1.5 & 108 \\
S6 & 3.5 & 2.32 & 2.93 & 2.85 & 3 \\
S7 & 3.4 & 2.56 & 2.97 & 2.96 & 0.3 \\
\hline
\end{tabular}

It is obvious that only samples \#2,\#6 and \# 7 have $k_{\text {eff }}$ with less than $5 \%$ deviations from the measured values. Please note that we will omit samples \#4 and \#5 from the discussion because both have quite high \% error deviations that exceeded $53 \%$ and $100 \%$, respectively. Furthermore, the measured data for \# 4 and \# 5 samples were not within the range of the estimated extreme Wiener bounds for maximum and minimum values of the constituents' conductivities.

Therefore, sample \#4 and sample \#5 do not satisfy the imposed assumption by the model; the necessary condition that is the existence of statistically homogeneous medium surrounding inclusions of different phases and they are out of the range of Wiener bounds.

More details about the volume fractions, compositions and the conductivities of phases of the samples are given elsewhere [39-40].

The apparent features (pictures and images) of the other five selected samples that were within the imposed assumptions are shown in Fig. 2.
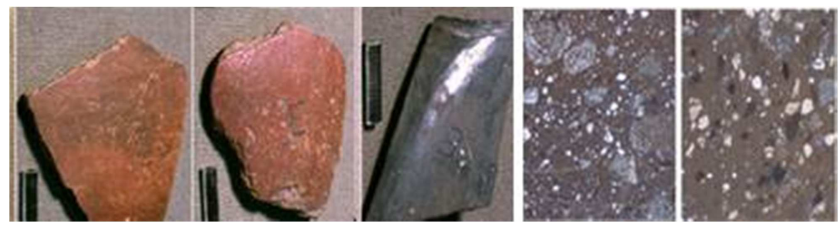

Figure 2. Typical samples three ceramic glasses and two marbles, showing the main apparent features including the shape and color of the foliations.
These five chosen samples have relatively low \% error ranging from 3 to $30 \%$. It seems that they satisfy the necessary condition of medium homogeneity with varying degrees. Samples \#6 and \#7 are both ceramics marbles with uniform morphology structure as shown by the last pictures in figure 2. The volumetric fractions of the mineral constituents were determined by the point-counting method using transparent thin slices, each having an area of $20 \times 30$ $\mathrm{mm}^{2}$ and an approximate thickness of $30 \mu \mathrm{m}$. The slices under the microscope showed an excellent level of uniform distribution of phases in these slides consistent with their images. The error deviation is less than $3 \%$ in these two marble samples. Although, we did not take into account possibilities of anisotropy of thermal conductivity of the selected samples, it seems that, assuming isotropic and homogeneous conditions, the thermal conductivity of such samples may be calculated using EMTM with rather good accuracy, within $\pm 3 \%$. This is in agreement with surface distribution profile in the slices and images of Fig 3.
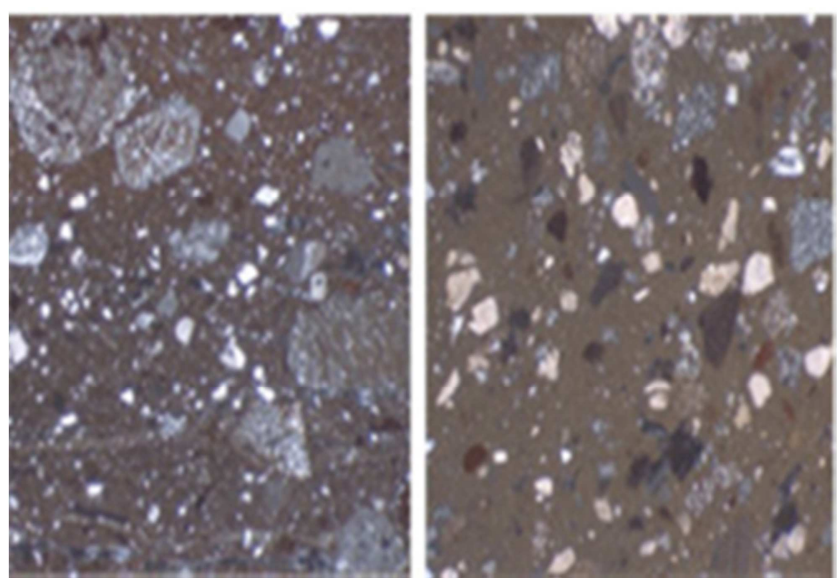

Figure 3. The homogeneity and uniform distribution of foliations within marble samples are very obvious form the apparent shape.

The analysis of scanning electron microscopy images for samples \#1, \#2 and \#3 are depicted in figure $4(\mathrm{a} \rightarrow \mathrm{c})$ with 
100 and 5000 magnifications. It is clear that the phase morphology for sample \# 2 has good uniform distribution of phases (Fig. 4a) while it is not the same level for samples \#1 \& \#3 as shown in Fig, 4b \& Fig. 4c.
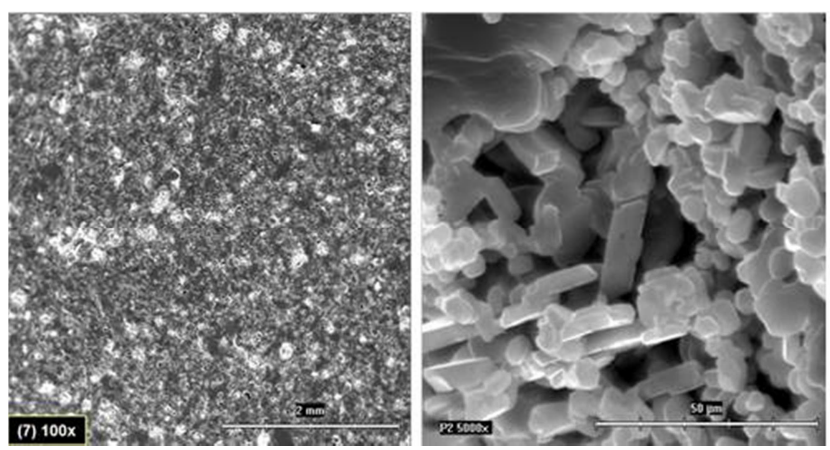

Figure 4a. Images of Sample \# 2 with less than 5\% deviation.
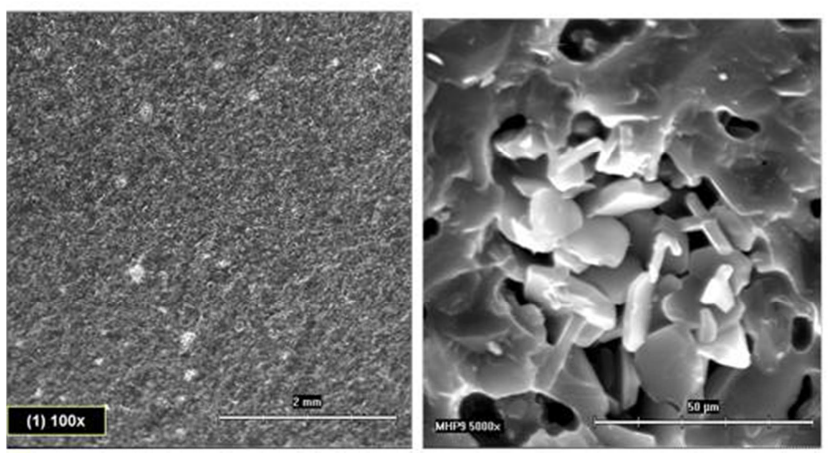

Figure 4b. Images of Sample \# 1 with 28\% deviation.

Note that Samples \# 1 \& \#3 have similar composition but different thermos-physical properties due to their detailed microstructure spatial distributions.
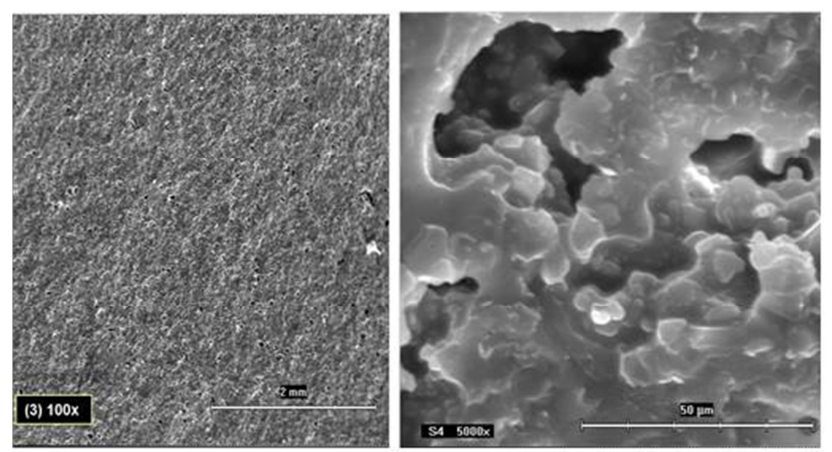

Figure 4c. Images of Sample \# 3 with 30\% deviation.

Thus, among the several theoretical models for estimating $k_{\text {eff }}$ in thermal conduction of composites, the tested model for the effective medium theory (EMTM), along with Wiener bounds, can be used to estimate the effective conductivity of macroscopic homogeneous and isotropic multiphase composite materials. We used Wiener bounds, as a preliminary indicator to validate the bulk homogeneity condition of the investigated samples, i.e. the measured values for the conductivity should be within the limits of the Wigner bounds and we used electron and/or optical microscope scanning to validate the surface homogeneity condition.

In other words, this model can be effectively used after determining the parallel and serial bounds (Wigner bounds) of the overall conductivity of the random mixture and after satisfying the surface homogeneity condition through simple and preliminary microstructure surface measurements.

\section{Conclusions}

In this class of composite ceramics, after a comparison between the experimental data of the effective thermal conductivity with the corresponding theoretical estimation, it turns out that the EMT model is a suitable one to estimate $k_{\text {eff }}$ provided that the homogeneity condition is satisfied.

Numerical calculated values of thermal conductivity for the selected samples that satisfy the homogeneity condition imposed by the effective medium theory are in best agreement with the experimentally measured ones. It was more suitable for samples \#6, \#7 and \#2 due to the fulfilment of Wiener bounds and enhanced uniformity in the distribution of phases determined by either the point-counting method using transparent thin slices or the STM for sample \#2.

It is worth to mention that there are a number of physical situations in which we have a linear relation(in our case) between a field e.g. the temperature field $\Delta \mathrm{T}$ and a flow, e.g. the heat current $Q$. In such situations, the constant of proportionality which is a material property (in our case is thermal conductivity), is generally a tensor but reduces to a scalar when the material is isotropic. This includes similar cases of estimating effective values of material properties such as electrical conductivity, dielectric permittivity, and magnetic permeability etc.

Therefore, the validation procedures implemented in this work may save effort, time and cost required to estimate and predict the desired properties for similar several applications that involves, thermal, electrical, magnetic and diffusion process. It may also help to improve the choice of the required composites and reduce the risk of possible failure during real time operation.

Although, we did not take into account possibilities of anisotropy of thermal conductivity of the selected samples, it seems that, assuming isotropic and homogeneous conditions, the thermal conductivity of such samples may be calculated using EMTM with rather good accuracy $( \pm 3 \%)$. It is worth mentioning that there are constrains imposed upon using these models such as limiting the analysis to room temperature, limiting the number of samples and neglecting the anisotropy in the thermal conductivities of the phases within the samples.

\section{Acknowledgements}

I would like to acknowledge Prof. Vlastimil Boháč and his group, at the Institute of physics, Slovak Academy of Sciences Bratislava, for providing the SEM images. 


\section{References}

[1] M. Zhang, P. C. Zhai, Y. Li, J. T. Zhang, Q. J. Zhang, "Effective Thermal Conductivity of Particulate Composites with Different Particle Configurations" AIP Conf. Proc. 973, 2008, p141.

[2] S.-Y. Zhao, B.-M. Zhang, X.-D. He, "Temperature and pressure dependent effective thermal conductivity of fibrous insulation Int. J. Therm. Sci. 48(2), 2009, p 440.

[3] L. Chaffron, C. Sauder, C. Lorrette, L. Briottet, A. Michaux, L. Gélébart, A. Coupé, M. Zabiego, M. Le Flem, And J.-L. Séran, "Innovative $\mathrm{SiC} / \mathrm{SiC}$ Composite for Nuclear Applications" EPJ Web of Conferences 51, 2013, 01003.

[4] F. Brigaud, D. S. Chapman, S. Le Douaran, "Estimating thermal conductivity in sedimentary basins using lithologic ..." AAPG Bull. 74, 1990, P1459.

[5] A. Correia, F. W. Jones, A. Fricker, "Terrestrial heat - flow density estimates for the Jeanne D'Arc Basin, offshore eastern Canada" Geophysics 55, 1990, P 1625.

[6] S. Suresh \& A. Mortensen, "Fundamentals of Functionally Graded Materials"IOM Communication” Ltd., London. 1998.

[7] Y. Xu \& K. Yagi “Automatic FEM model generation for evaluating thermal conductivity of composite with random materials arrangement" Comput. Mater. Sci. 30 2004, P242252.

[8] S.J. Zinkle \& L.L. Snead "Thermophysical and Mechanical Properties of $\mathrm{SiC} / \mathrm{SiC}$ " Composites an upcoming version of the fusion Materials Properties Handbook (MPH) J.W. Davis ed. (Boeing, St. Louis). Oak Ridge National Laboratory, 1999.

[9] O.Wiener," Abhandlungen der Mathematish-Physischen Klasse der K6niglichen Sachsischen Gesellschaft der Wissenschaften”, Leipzig, 1912, p. 509.

[10] B. M Suleiman1, S. E. Gustafsson1, E. Karawacki1, R. Glamheden' and U. Lindblom', 'Effective Thermal Conductivity of Amulti-Phase Sys'iem" Journal of thermal Analysis, Vol. 51, 1998.

[11] (a) M. Koizumi "The concept of FGM, in: J. B. Holt, M. Koizumi, T. Hirai, Z. A. Munir (Eds.)," Ceramic Transactions, Functionally Graded Materials", vol. 34, The American Ceramic Society, 1993.

[12] (b) M. Koizumi, M. Niino, Overview of FGM Research in Japan, MRS Bulletin, 1 1995, p 19-21.

[13] X.-Q, Fang J-X LIU \& T. Zhang "Dynamic effective thermal properties of functionally graded. fibrous composites using non-Fourier heat conduction Computational" Journal of Composite Materials Vol. 43, No. 21, 2009, P2351-2369.

[14] Gray L. J., Kaplan T. \& Richardson J. D., Paulino G. H., and Mem. ASME "Green's Functions and Boundary Integral Analysis for Exponentially Graded Materials: Heat Conduction" J. Appl. Mech. 70 2003,-P543.

[15] Kuo H.-Y. \& Chen T. "Steady and transient Green's functions for anisotropic conduction in an exponentially graded solid". Int. J. Solids Struct. 42: 2005, P1111-1128.

[16] Cahill D. G., Braun Paul V., Chen G., Clarke D. R., Fan S., Goodson K. E., Keblinski P., King W. P., Mahan G. D.,
Majumdar A., Maris H. J., Phillpot S. R., Pop E., and Shi Li," Nanoscale thermal transport II" Applied Physics Reviews 1, 2014, 011305.

[17] J. C.Maxwell (1892), A Treatise on Electricity and Magnetism, third ed., vol. 1, Dover, NewYork. Oxford: Clarendon 1954, p 435-41.

[18] Rayleigh, L. On the influence of obstacles arranged in rectangular order upon the properties of the medium. Philosophical Magazines 34 481-502, 1892.

[19] D. Polder \& J H Van Santen" The effective permeability of mixtures of solids "Physica 12 257-71, 1946.

[20] A. Baldan, "Progress in Ostwald Ripening Theories and Their Applications to Nickel-Base." Journal of Materials Science 37 2002, p2171-2202.

[21] J. A. Reynolds \& J. M. Hough "Formulae for Dielectric Constant of Mixtures" Proc. Phys. Soc. B 70, 1957, P769-75.

[22] R. L. Hamilton \& O. K. Crosser, "Thermal Conductivity of Heterogeneous Two-Component Systems" Ind. Eng. Chem. Fundamen., 1 (3), 1962, pp 187-191.

[23] A Rocha \& A Acrivos "Experiments on the Effective Conductivity of Dilute Dispersions Containing Highly Conducting Slender Inclusions" Proc. R. Soc. A 337, 1974, P123-33.

[24] R. McPhedran \& D. McKenzie "The conductivity of lattices of spheres. in the simple cubic lattice" Proceeding of the Royal Society of London: A 359 1978, P45-63.

[25] .A. Sangani \& A. Acrivos "Effective Conductivity Of A Periodic Array of Spheres" Proceeding of the Royal Society of London: A 386 1983, P263-275.

[26] Z. Hashin "Assessment of the self-consistent scheme approximation: conductivity of particulate composites" Journal of Composite Materials 2 1968, 284-300,

[27] Y. Benveniste \& T. Miloh" On the effective thermalconductivity of coated short-fiber composites." Journal of Applied Physics 69, 1991, 1337-1344.

[28] J.D. Felske "Effective thermal conductivity of composite spheres in a continuous medium with contact resistance, Int. Heat Mass Transfer 47 2004, p 3453-3461.

[29] P. K. Samantray, P. Karthikeyan \& K. S Reddy. "Estimating effective thermal conductivity of two-phase materials." International Journal of Heat and Mass Transfer 49, 2006, P 4209-4219.

[30] Jinzao Xu, Benzheng Gao, Hongda Du, Feiyu Kang “A statistical model for effective thermal conductivity of composite materials" International Journal of Thermal Sci., 104, 2016, P 348-356.

[31] T W Noh, P H Song \& A J Sievers "Self-consistency conditions for the effective-medium approximation in composite materials: Phys. Rev. B 44 1991, P 123-165.

[32] G. W. Milton, "The coherent potential approximation is a realizable effective medium scheme" Commun. Math. Phys., 99 1985, P463.

[33] L. Tan, "Multiple Length and Time-scale Approaches in Materials Modeling", Advances in Materials Volume 6, Issue 1-1, (2017), P 1-9. 
[34] S.Tang, B. Zhu, M. Jia, Q. He, S. Sun, Y. Mei, and L. Zhou, "Effective-medium theory for one-dimensional gratings" Phys. Rev. B 91, 2015, P174201.

[35] J.-B. Vaney, A. Piarristeguyb, V. Ohorodniichucka, O. Ferry, A. Pradel, E. Alleno, J. Monnier, E. B. Lopes, A. P. Gonçalves, G. Delaizir, C. Candolfi, A. Dauschera and B. Lenoira "Effective medium theory based modeling of the thermoelectric properties of composites: comparison between predictions and experiments in the glass-crystal composite system $\mathrm{Si}_{10} \mathrm{As}_{15} \mathrm{Te}_{75}-\mathrm{Bi}_{0.4} \mathrm{Sb}_{1.6} \mathrm{Te}_{3}$ ", J. Mater. Chem. C, 3, 2015, P11090-11098.

[36] T H. Jordan, "An effective medium theory for threedimensional elastic heterogeneities" Geophysical Journal International Volume 203, Issue 2, 2015, P. 1343-1354.
[37] M. Siddiqui and A. M. Arif, "Generalized Effective Medium Theory for Particulate Nanocomposite Materials", Materials, 9, 2016, P694.

[38] http://www.compositesworld.com/articles/composites-2016materials-and-processes.

[39] G. Angenheister (ed.), Landolt-Börnstein, Numerical Data and Functional Relationships in Science and Technology, vol. 1/a (Springer, Berlin, 1982, p. 311.

[40] B. M. Suleiman, "Effective Thermal Conduction in Composite Materials" Applied Physics A, Material Sciences \& Processing, 2010, 99 P223-228. 